\title{
Garenoxacin is effective against bacteria invading cells such as Streptococcus pyogenes and Haemophilus influenzae
}

Hiroyuki luchi ( $\sim$ hamoshun@m.kufm.kagoshima-u.ac.jp )

Kagoshima University

Junichiro Ohori

Kagoshima University

Satoshi Kiyama

Kagoshima University

\section{Research Article}

Keywords: antibacterial drugs, Haemophilus influenzae, Streptococcus pyogenes, susceptibility, garenoxacin

Posted Date: February 10th, 2021

DOI: https://doi.org/10.21203/rs.3.rs-152395/v1

License: (9) This work is licensed under a Creative Commons Attribution 4.0 International License. Read Full License 


\section{Abstract}

Background: Recurrent tonsillitis is one of the most common otolaryngological disorders caused by bacteria invading cells such as Streptococcus pyogenes (S. pyogenes) and Haemophilus influenzae. The aim of this study was to investigate the effect of antibacterial drugs against bacteria that have invaded cells.

Results: The intracellular invasion of Detroit 562 cells by five strains of nontypeable Haemophilus influenzae (NTHi) and four strains of Streptococcus pyogenes was investigated. The antibacterial drugs used were garenoxacin (GRNX), clarithromycin (CAM), amoxicillin (AMPC), cefditoren pivoxil (CDTR-PI), and levofloxacin (LVFX). Both NTHi and S. pyogenes fully invaded Detroit 562 cells in 6 h. Both NTHi and S. pyogenes had reduced susceptibility to CAM. GRNX, CAM, and LVFX were effective against bacteria invading the cells, but AMPC and CDTR-PI were not effective. GRNX was the most effective.

Conclusion: GRNX was the most effective agent against bacteria invading cells.

\section{Background}

Recurrent tonsillitis is one of the most common otolaryngological disorders [1]. The most frequent cause is viruses, and the second most frequent cause is bacteria, such as Streptococcus pyogenes ( $S$. pyogenes), Staphylococcus aureus, Haemophilus influenzae, and Streptococcus pneumoniae [2]. Recurrent tonsillitis is caused by nontypeable $H$. influenzae (NTHi) and $S$. pyogenes that enter the cells and escape from the action of antibacterial drugs [1]. Tonsillitis that is not cured by initial treatment requires a change of antibacterial agents or the selection of agents against bacteria that have invaded the cells.

H. influenzae is a leading cause of acute and chronic otitis media, chronic sinusitis, and tonsillitis [3]. It is reported that in otitis media and chronic sinusitis, most strains of $H$. influenzae lack capsular polysaccharides and are referred to as nontypeable $H$. influenzae (NTHi), and that $H$. influenzae bacteria frequently persist within dense biofilm communities that are thought to provide resistance to host clearance and bactericidal activity of some antibiotics [4]. S. pyogenes is an important human pathogen that can cause severe, life-threatening, invasive infections such as soft-tissue infection, sepsis, and streptococcal toxic shock syndrome [5]. S. pyogenes is generally an extracellular pathogen that can survive and persist within the host by the expression of a broad array of virulence functions directed to circumventing the host immune mechanisms [6]. It is considered that recurrent tonsillitis is caused by NTHi and S. pyogenes entering the cells and escaping from the action of antibacterial drugs.

International guidelines recommend penicillin as the first-choice antibiotic treatment for acute sore throat (suspected to be caused by S. pyogenes) [7]. However, a recent meta-analysis of clinical studies reported that cephem drugs are more effective than penicillin drugs [8] and are effective as short-term therapy [9]. Therefore, it has become necessary to reconsider the conventional treatment policy based on penicillin. Beta-lactamase-negative ampicillin-resistant $H$. influenzae (BLNAR) is particularly common in 
Japan [10]. Therefore, tonsillitis that is not cured by initial treatment requires a change of antibacterial agents or the selection of antibacterial agents against bacteria that have invaded the cells.

Levofloxacin (LVFX), a broad-spectrum fluoroquinolone with potent activity against gram-positive bacteria, is currently recommended to treat respiratory tract infections and pneumonia due to $S$. pneumoniae, one of the most important causative pathogens in community-associated pneumonia (CAP). Similarly, garenoxacin (GRNX) is an oral des fluoro (6)-quinolone with potent antimicrobial activity against common respiratory pathogens [11]. LVFX and GRNX show similar antimicrobial activities against gram-negative bacteria. However, GRNX has higher antimicrobial activity than LVFX against gram-positive bacteria, including staphylococci, streptococci, and pneumococci [12]. Additionally, GRNX has higher broad-spectrum antimicrobial activity against anaerobes than LVFX [13]. These data suggest that GRNX may be an attractive agent for the treatment of CAP.

Clarithromycin (CAM) exerts its antibacterial activity through its inhibitory effect on protein synthesis and is therefore effective against atypical pathogens such as Mycoplasma pneumoniae and Chlamydia pneumoniae that do not have cell walls [14]. It also has antibacterial activity against intracellular parasites such as Legionella and nontuberculous mycobacteria, reflecting its excellent transferability from tissues to cells [15].

The present study investigated the in vitro antibacterial activity of antibacterial agents against clinical strains of NTHi and S. pyogenes isolated in Japan.

\section{Materials And Methods}

\section{Antibacterial agents}

The following antibacterial agents were used in the study: analytical grade powders of GRNX (FUJIFILM Toyama Chemical Co., Ltd., Tokyo, Japan), CAM (Meiji Seika Pharma, Tokyo, Japan), amoxicillin (AMPC) (Wako Pure Chemical Industries), cefditoren pivoxil (CDTR-PI) (Meiji Seika Pharma, Tokyo, Japan), and LVFX (Sigma-Aldrich, Tokyo, Japan).

\section{Bacteria and growth conditions}

We evaluated five clinical strains of NTHi isolated from the nasopharynges of patients with otitis media with effusion and four clinical strains of $S$. pyogenes isolated from tonsillar crypts of patients with recurrent tonsillitis. All bacteria were stored in skimmed milk with glycerol at $-80^{\circ} \mathrm{C}$ until use. An aliquot of each bacterial stock was thawed and cultured overnight at $37^{\circ} \mathrm{C}$ in a $5 \% \mathrm{CO}_{2}$ incubator on chocolate II agar (Nippon Becton Dickinson Co. Ltd., Tokyo, Japan) or sheep blood agar (Nissui Pharmaceutical Co., Ltd., Tokyo, Japan) plates, as appropriate. After washing in $0.5 \%$ bovine serum albumin-phosphatebuffered saline (PBS), the bacteria were used for intracellular invasion assays. The concentrations of NTHi and S. pyogenes were adjusted to $1.0 \times 10^{8}$ colony-forming units (CFU)/mL at an absorbance of $580 \mathrm{~nm}$. This study was approved by the Institutional Review Board of Kagoshima University 


\section{Determination of minimum inhibitory concentration (MIC)}

The susceptibility of bacteria to antibiotics was studied by the broth microdilution method, performed according to Clinical Laboratory Standards Institute guidelines [15]. The test medium was prepared using cation-adjusted Mueller Hinton broth (Eikenkagaku, Tokyo, Japan) with lysed horse blood (Nippon Biotest Laboratory, Tokyo, Japan). The quinolones evaluated were GRNX, CAM, AMPC, CDTR-PI, and LVFX. In this study, 1 and 2 MIC were used.

\section{Cell culture}

Detroit 562 cells (CCL-138; ATCC, Manassas, VA, USA), a human pharyngeal carcinoma epithelial cell line, were grown to confluence in minimal essential medium (Nacalai Tesque Inc., Kyoto, Japan) supplemented with $1 \mathrm{mM}$ sodium pyruvate (Nacalai Tesque), 10\% fetal bovine serum (Invitrogen, San Diego, CA, USA), penicillin $(100 \mathrm{U} / \mathrm{mL})$, and streptomycin $\left(100 \mu \mathrm{g} / \mathrm{mL}\right.$; Nacalai Tesque) at $37^{\circ} \mathrm{C}$ in a $5 \%$ $\mathrm{CO}_{2}$ incubator as previous described [16]. The cells were harvested using trypsin (final concentration, $0.02 \%$ ) and ethylenediaminetetraacetic acid (EDTA; final concentration, $0.02 \%$; Nacalai Tesque) and seeded at a density of $2 \times 10^{4}$ viable cells per well in a 96-well BD Falcon tissue culture plate with a lowevaporation lid (BD Biosciences, Franklin Lakes, NJ, USA). The plates were used when $>90 \%$ confluence was observed following overnight incubation.

\section{Intracellular invasion assay}

One hundred microliters each of the NTHi and S. pyogenes strains $\left(1.0 \times 10^{8} \mathrm{CFU} / \mathrm{mL}\right)$ were added to Detroit 562 cells cultured in a 96 -well plate and allowed to adhere at $37^{\circ} \mathrm{C}$ in a $5 \% \mathrm{CO}_{2}$ incubator for 6 h. Each well was then treated with gentamicin $(200 \mu \mathrm{g} / \mathrm{mL})$ at $37^{\circ} \mathrm{C}$ in a $5 \% \mathrm{CO}_{2}$ incubator for $1 \mathrm{~h}$. After washing five times with $200 \mu \mathrm{L}$ of PBS, the cells were treated with $100 \mu \mathrm{L}$ of each antibacterial agent at $37^{\circ} \mathrm{C}$ in a $5 \% \mathrm{CO}_{2}$ incubator for $6 \mathrm{~h}$. After washing five times with $200 \mu \mathrm{L}$ of PBS, the cells were treated with $100 \mu \mathrm{L}$ of saponin at $37^{\circ} \mathrm{C}$ in a $5 \% \mathrm{CO}_{2}$ incubator for $15 \mathrm{~min}$. Further, $100 \mu \mathrm{L}$ of the samples from each well was plated on chocolate II agar plates or sheep blood agar and cultured overnight, and the number of colonies formed was counted as previous decribed [17].

\section{Statistical analysis}

All values are presented as means \pm standard deviation. The data were statistically analyzed using the unpaired one-way ANOVA with Tukey's method. We considered differences to be statistically significant when the probability values were $<5 \%$.

\section{Results}

\section{Bacterial invasion time}


NTHi bacteria were found inside the cells $2 \mathrm{~h}$ after they were attached to the cells (Fig. 1A). After 4 and $6 \mathrm{~h}$, the number of bacteria invading the cells increased in a time-dependent manner (Fig. 1A). However, there was no difference between the numbers of bacteria invading the cells at $6 \mathrm{~h}$ and $8 \mathrm{~h}$ (Fig. 1A). Similarly, the number of $S$. pyogenes bacteria invading the cells increased in a time-dependent manner, and there was no difference between the numbers of bacteria invading the cells at $6 \mathrm{~h}$ and $8 \mathrm{~h}$ (Fig. 1B). Based on these results, the time to enter the cells was set to $6 \mathrm{~h}$.

\section{Minimum inhibitory concentration}

In invasion by NTHi, no reduction in susceptibility was observed with GRNX, AMPC, and LVFX. However, AMPC and especially CAM showed decreased sensitivity (MIC $\geq 2 \mu \mathrm{g} / \mathrm{mL}$ ). As with $\mathrm{NTHi}, S$. pyogenes did not show a decrease in sensitivity to GRNX, AMPC, and LVFX. However, LVFX showed decreased sensitivity (MIC $\geqq 0.5 \mu \mathrm{g} / \mathrm{mL}$ ) (Table 1 ).

\section{Effects of antibacterial agents on NTHi}

Cells invaded by bacteria, treated with saponin, and then treated with PBS served as a control. Treatment with 1 MIC of GRNX, CAM, or LVFX significantly reduced the number of NTHi bacteria entering the cells (Fig. 2A) $(p<0.05)$. GRNX had a significantly higher bactericidal effect than CAM and LVFX ( $p<$ 0.05). However, no bactericidal effect was observed from treatment with AMPC or CDTR-PI (Fig. 2A).

Similarly, treatment with 2 MIC of GRNX, CAM, or LVFX also had a significant bactericidal effect ( $p$ $<0.05$ ), but treatment with AMPC or CDTR-PI had no bactericidal effect (Fig. 2B). GRNX had the highest bactericidal effect (Fig. 2B).

\section{Effects of antibacterial agents on S. pyogenes}

Cells invaded by bacteria, treated with saponin, and then treated with PBS served as a control. Treatment with 1 MIC of GRNX, CAM, or LVFX significantly reduced the number of $S$. pyogenes bacteria entering the cells (Fig. 3A) $(p<0.05)$. GRNX had a significantly higher bactericidal effect than CAM and LVFX (Fig. 3A) $(p<0.05)$. However, no bactericidal effect was observed from treatment with AMPC or CDTR-PI (Fig. 3A).

Similarly, treatment with 2 MIC of GRNX, CAM, or LVFX also had a significant bactericidal effect ( $p$ $<0.05$ ), but treatment with AMPC or CDTR-PI had no bactericidal effect (Fig. 3B). GRNX had the highest bactericidal effect (Fig. 3B).

\section{Discussion}

This study investigated the effects of GRNX, CAM, AMPC, CDTR-PI, and LVFX on the invasion of Detroit 562 cells by NTHi and S. pyogenes. The results showed that NTHi and S. pyogenes invaded Detroit 562 cells, AMPC and CDTR-PI did not affect the invasion of Detroit 562 cells by NTHi and S. pyogenes, and GRNX, CAM, and LVFX reduced the invasion of Detroit 562 cells by NTHi and S. pyogenes. 
Fibronectin-binding protein (F1 protein) is mentioned as a mechanism by which $S$. pyogenes invades the cells [18]. In Japan, Ma et al. [19] reported that $77.3 \%$ of $S$. pyogenes strains possessed F1 protein. More interestingly, many biofilm-producing strains are F1 protein-negative strains [20]. Intracellular invasion ability and biofilm formation ability are negatively correlated, and it is considered that $S$. pyogenes avoids the attack of antibacterial drugs [18]. In addition, phosphorylcholine is mentioned as a mechanism of intracellular invasion by NTHi, and the higher the expression level of phosphorylcholine, the more it penetrates into cells [17]. The present study showed that it takes a certain period of time for bacteria to adhere to cells and enter the cells, which becomes constant within 6 h. Yamanaka's report that $H$. influenzae invades Detroit 562 cells supports the results of the present study [21].

Since the late 1990s, respiratory tract infections caused by resistant strains of $S$. pneumoniae and $H$. influenzae have been rapidly increasing worldwide. Penicillin-resistant S. pneumoniae, such as penicillin intermediately-resistant S. pneumoniae(PISP), penicillin-resistant S. pneumoniae (PRSP), and BLNAR, are particularly common in Japan [10]. In this study, no bacteria resistant to $\beta$-lactam were found, but decreased sensitivity to LVFX by $S$. pyogenes and to CAM by NTHi and $S$. pyogenes was observed. Quinolone inhibits bacterial growth by disrupting the DNA replication of type II topoisomerase [22]. Type II topoisomerases are currently recognized to include DNA gyrase, which is responsible for the formation and elimination of supercoiled structures in DNA strands, and topoisomerase IV, which cuts and re-ligates tangled DNA during DNA replication [22]. Both of these enzymes are composed of two dimers of subunit types $A$ and $B$, which together form a tetramer. Amino acid substitutions in either enzyme may lead to the inhibition of such complex formations, and in particular, mutations in the quinolone resistancedetermining regions (QRDRs) within subunits A and B are closely related to resistance [23]. Shoji et al. [24] reported that of the 14 S. pyogenes strains, 12 (85.7\%) had two or more mutations in QRDRs. This is considered one of the reasons that streptococcal susceptibility to LVFX was reduced.

Invasion of cells by bacteria has been cited as a cause of repeated tonsillitis. In this study, neither AMPC nor CDTR-PI was found to have a bactericidal effect on bacteria invading the cells. It is known that $\beta$-lactam antibacterial drugs have low intracellular transmissibility, and their antibacterial action is reduced against $H$. influenzae that has entered the cells [24]. Therefore, it is suggested that another antimicrobial treatment is necessary for recurrent tonsillitis.

GRNX shows a favorable pharmacokinetic profile, with good penetration into sputum and otorhinolaryngological tissues, and it is highly effective in the treatment of patients with upper and lower respiratory tract infections [11]. Recent studies have established that the $\mathrm{AUC}_{0-24} / \mathrm{MIC}$ ratio is an important pharmacodynamic parameter influencing quinolone efficacy. Lister demonstrated that garenoxacin exhibits pharmacodynamics similar to those of clinically available quinolones [25]. In addition, Takagi et al. [26] reported that GRNX concentrations in plasma and tissues of subjects receiving GRNX 400 mg once a day were higher than the $\mathrm{MIC}_{90}$ of major causative pathogens. The trough concentration (Cmin) in plasma was $1.92 \mathrm{~g} / \mathrm{mL}$, a level that was higher than the mutant prevention concentration, suggesting that GRNX is unlikely to induce the selection of resistant strains during 
treatment. The efficacy rates of GRNX in otorhinolaryngological infections were $91.3 \%$ for sinusitis, $81.8 \%$ for otitis media, $89.5 \%$ for pharyngolaryngitis, and $95.0 \%$ for tonsillitis [26]. A double-blind study was conducted comparing GRNX $400 \mathrm{mg}$ once a day with LVFX $100 \mathrm{mg}$ three times a day for 10 days in patients with bacterial pneumonia. The efficacy rate was $94.9 \%$ (94/99) in the GRNX group and $92.8 \%$ $(77 / 83)$ in the LVFX group at the 7th day after completion of treatment [27]. No significant difference in efficacy rates was found between GRNX and LVFX, with a $95 \% \mathrm{Cl}$ of $-4.9 \%$ to $9.2 \%$, indicating that GRNX is not inferior to LVFX. The bacterial eradication rate was $100 \%(53 / 53)$ in the GRNX group and $87.8 \%$ $(36 / 41)$ in the LVFX group. This difference in the eradication rate was statistically significant, with a $95 \%$ $\mathrm{Cl}$ of $2.4 \%$ to $23.9 \%$ [27]. In the present study, GRNX was also effective against bacteria invading cells. Since LVFX showed decreased sensitivity, GRNX is effective for recurrent tonsillitis.

The present study showed that CAM was effective against bacteria invading cells. Patel et al. reported that the concentration of CAM in alveolar macrophages of healthy subjects reached a maximum of $1996 \mu \mathrm{g} / \mathrm{mL}$ at $4 \mathrm{~h}$ after administration of $500 \mathrm{mg}$ of CAM [28]. Chou et al. [29] reported that cultured human gingival fibroblasts and SCC-25 cells took up CAM via a concentrative active transport system. However, the concentration of CAM used in this study is far beyond the amount used in actual clinical practice and therefore could not be used in actual clinical practice.

Our study has some limitations. First, BLNAS and other resistant strains were not investigated. Since the number of strains of resistant bacteria is increasing, more resistant strains should be included in future studies. The second limitation pertains to the epithelial cells used. Although the use of normal human epithelial cells may be more clinically relevant, we used a pharyngeal cancer-derived cell line. Because these cells were of human origin, we consider that the results of this study were not different from those that would have been obtained with the use of normal cells.

In conclusion, GRNX was the most effective agent against bacteria invading cells. Administration of GRNX should be considered when the efficacy of penicillin and cephem antibiotics and of $\beta$-lactam is insufficient in daily medical practice.

\section{Declarations}

\section{Availability of data and materials}

The datasets used and analyzed during the current study are available from the corresponding author on reasonable request.

\section{Acknowledgements}

We thank Ms. S. Katahira for her technical assistance in our laboratory. We thank the members of our laboratory for their technical advice and discussions.

\section{Funding}


This work was supported by JSPS KAKENHI Grant Number 20K18258.

\section{Ethics declarations}

\section{Ethics approval and consent to participate}

We obtained written informed consent all subjects or, if subjects are under 18, from a parent or legal guardian. This study was approved by Kagoshima University ethics committee (200313).

All methods were carried out in accordance with relevant guidelines and regulations.

\section{Consent for publication}

Not applicable.

\section{Competing interests}

The authors declare that they have no conflict of interest.

\section{Contributions}

Hiroyuki luchi: methodology, formal analysis, and writing the original draft. Junichiro Ohori: software, investigation and validation. Satoshi Klyama: resources, visualization, project administration, data curation, conceptualization, supervision. All authors read and approved the final manuscript.

\section{References}

1. Loganathan A, Arumainathan UD, Raman R. Comparative study of bacteriology in recurrent tonsillitis among children and adults. Singapore Med J 2006;47:271.

2. Gul M, Okur E, Ciragil P, Yildirim I, Aral M, Akif Kilic M. The comparison of tonsillar surface and core cultures inrecurrent tonsillitis. Am J Otolaryngol 2007;28:173-6.

3. Hall-Stoodley L, Hu FZ, Gieseke A, Nistico L, Nguyen D, Hayes J, et al. Direct detection of bacterial biofilms on the middle-ear mucosa of children with chronic otitis media. JAMA 2006;1296:202-11.

4. Bakaletz LO. Bacterial biofilms in otitis media: evidence and relevance. Pediatr Infect Dis J 2007;26:S17-9.

5. Efstratiou A. Group A streptococci in the 1990s. J Antimicrob Chemother 2000;45:3-12.

6. Raeder R, Boyle MDP. Association of type II immunoglobulin G-binding protein expression and survival of group A streptococci in human blood. Infect Immun 1993;61:3696-702.

7. Matthys J, De Meyere M, van Driel ML, De Sutter A. Differences among international pharyngitis guidelines: not just academic. Ann Family Med 2007;5:436-43.

8. Casey JR, Pichichero ME. Meta-analysis of cephalosporin versus penicillin treatment of group A streptococcal tonsillopharyngitis in children. Pediatrics 2004;113:866-82. 
9. Casey JR, Pichichero ME. Meta-analysis of short course antibiotic treatment for group a streptococcal tonsillopharyngitis. Pediatric Infect Dis J 2005;24:909-17.

10. Suzuki K, Nishimura T, Baba S. Current status of bacterial resistance in the otolaryngology field: results from the Second Nationwide Survey in Japan. J Infect Chemother 2003;9:46-52.

11. Jones RN, Sader HS, Stilwell MG, Fritsche TR. Garenoxacin activity against isolates from patients hospitalized with community-acquired pneumonia and multidrug-resistant Streptococcus pneumonia. Diagn Microbiol Infect Dis 2007;58:1-7.

12. Fung-Tomc JC, Minassian B, Kolek B, Huczko E, Aleksunes L, Stickle T, et al. Antibacterial spectrum of a novel der-fluoro(6) quinolone, BMS-284756. Antimicrob Agents Chemother 2000;44:3351-6.

13. Hoellman DB, Kelly LM, Jacobs MR, Appelbaum PC. Comparative antianaerobic activity of BMS 284756. Antimicrob Agents Chemother 2001;45:589-92.

14. McCarty JM. Clarithromycin in the management of community-acquired pneumonia. Clin Ther 2000;22:281-94.

15. Cockerill FR, Wikler MA, Alder J, Dudley MN, Eliopoulos GM, Ferraro MJ, et al. Methods for dilution antimicrobial susceptibility tests for bacteria that grow aerobically; approved standard. 7th ed. Wayne: Clinical and Laboratory Standards Institute; 2006.

16. luchi H, Ohori J, Kyutoku T, Kotoko I, Kawabata M. Inhibitory effects of 2-methacryloyloxyethyl phosphorylcholine polymer on the adherence of bacteria causing upper respiratory tract infection. $J$ Oral Microbiol 2020;12(1):1808425

17. Iuchi H, Ohori J, Kyutoku T, Ito K, Kurono Y. Role of phosphorylcholine in Streptococcus pneumoniae and nontypeable Haemophilus influenzae adherence to epithelial cells. ANL 2019;46:513-9.

18. Jadoun J, Ozeri V, Burstein E, Skutelsky E, Hanski E, Sela S. Protein F1 is required for efficient entry of Streptococcus pyogenes into epitherial cells. J Infect Dis 1998;178:147-58.

19. Ma X, Kikuta H, Ishiguro N, Yoshioka M, Ebihara T, Murai, et al. Association of the prtF1 gene (encoding fibronectin-binding protein $\mathrm{F} 1$ ) and the sic gene (encoding the streptococcal inhibitor of complement) with emm types of group A streptococci isolated from Japanese children with pharyngitis. J Clin Microbiol 2002;40:3835-7.

20. Baldassarri L, Creti R, Recchia S, Imperi M, Facinelli B, Giovanetti, et al. Therapeutic failures of antibiotics used to treat macrolidesusceptible Streptococcus pyogenes infections may be due to biofilm formation. J Clin Microbiol 2006;44:2721-7.

21. Yamanaka N. Bactericidal effect of tosufloxacin on Haemophilus influenzae internalized in cultured human cells. Jpn J Antibiot 2016;69:309-18.

22. Shoji $\mathrm{H}$, Takuma T, Niki Y. Molecular analysis of levofloxacin-resistant Streptococcus pneumoniae in japan. Showa Univ J Med Sci 2014;2:181-90.

23. Hooper DC. Mechanisms of fluoroquinolone resistance. Drug Resist Updat 1999;2:38-55.

24. Hotomi M, Arai J, Billal DS, Takei S, Ikeda Y, Ogami M, et al. Nontypeable Haemophilus influenzae isolated from intractable acute otitis media internalized into cultured human epithelial cells ANL 
2010;37:137-44.

25. Lister P.D. Impact of $\mathrm{AUC}_{0-24} / \mathrm{MIC}$ ratios on the pharmacodynamics of the des-F(6) quinolone garenoxacin (BMS-284756) is similar to other fluoroquinolones. J Antimicrob

Chemother 2003;51:199-202.

26. Takagi H, Tanaka K, Hisatsugu T, Kobayashi H. Clinical studies of garenoxacin. Int J Antimicrob Agents2008;32:468-74.

27. Kobayashi H, Watanabe A, Aoki N, Odagiri S, Kawai S, Niki Y, et al. Clinical phase III comparative study on garenoxacin versus levofloxacin in patients with bacterial pneumonia. Nippon KagakuRyoho Gakkai Zasshi 2007;55:127-43.

28. Patel KB, Xuan D, Tessier PR, Russomanno JH, Quintiliani R, Nightingale $\mathrm{CH}$. Comparison of bronchopulmonary pharmacokinetics of clarithromycin and Antimicrob Agents Chemother 1996;40:2375-9》

29. CVhou CH, Walters JD. Clarithromycin transport by gingival fibroblasts and epithelial cells. J Dent Res 2008;87:777-81.

\section{Table}

Table 1. Minimum inhibitory concentrations of antibacterial agents against nontypeable Haemophilus influenzae and Streptococcus pyogenes

A

\begin{tabular}{llllll} 
strain & \multicolumn{5}{c}{ MIC $(\mu \mathrm{g} / \mathrm{ml})$} \\
\cline { 2 - 6 } & \multicolumn{5}{c}{ GRNXCAMAMPC CDTR LVFX } \\
\hline NTHi 1 & 0.06 & 4 & 0.5 & 0.06 & 0.06 \\
NTHi 2 & 0.06 & 4 & 0.5 & 0.06 & 0.06 \\
NTHi 3 & 0.06 & 8 & 0.5 & 0.06 & 0.06 \\
NTHi 4 & 0.06 & 2 & 0.5 & 0.06 & 0.06 \\
NTHi 5 & 0.06 & 4 & 0.5 & 0.06 & 0.06
\end{tabular}

B 


\begin{tabular}{lccccc}
\multicolumn{7}{c}{ GRNX CAMAMPC CDTR LVFX } \\
\cline { 2 - 6 } S. pyogenes 1 & 0.06 & 0.25 & 0.06 & 0.06 & 0.5 \\
S. pyogenes 2 & 0.06 & 16 & 0.06 & 0.06 & 0.5 \\
S. pyogenes 3 & 0.12 & 0.25 & 0.06 & 0.06 & 2 \\
S. pyogenes 4 & 0.12 & 0.25 & 0.06 & 0.06 & 0.5
\end{tabular}

MIC, minimum inhibitory concentration; NTHi, nontypeable Haemophilus influenzae;

GRNX, garenoxacin; CAM, clarithromycin; AMPC, amoxicillin; CDTR-PI, cefditoren pivoxil; LVFX, levofloxacin.

\section{Figures}

A

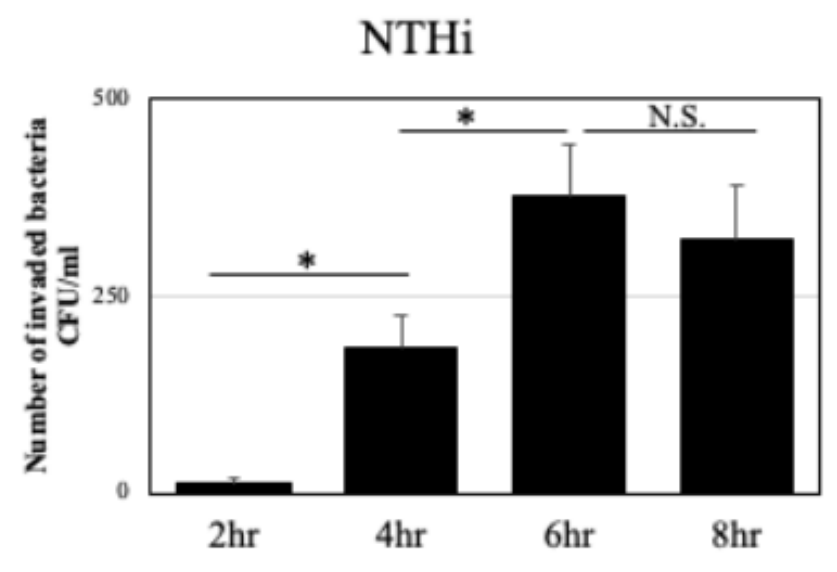

B

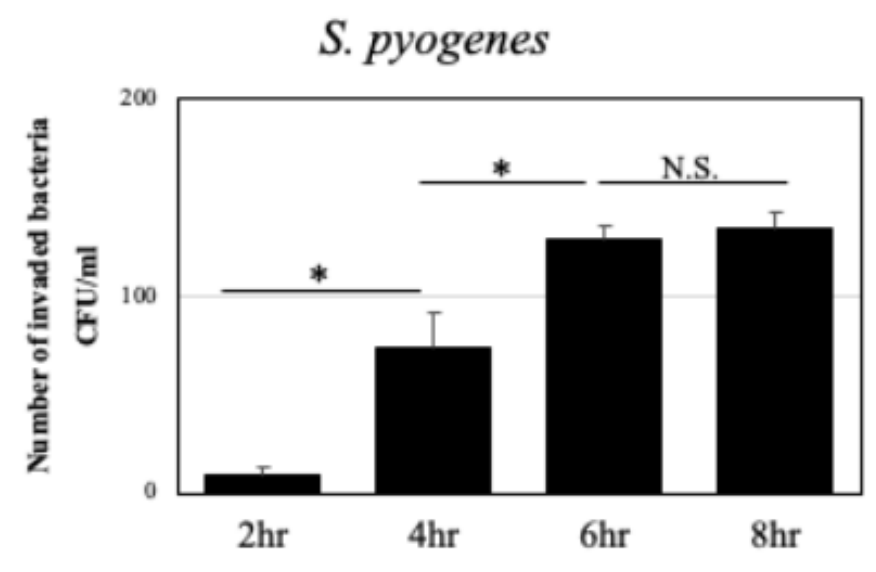

\section{Figure 1}

Bacterial invasion time Nontypeable Haemophilus influenzae (NTHi) entered the cells $2 \mathrm{~h}$ after adhered to the cells $\llbracket A \rrbracket$. The maximum invasion was at $6 \mathrm{~h} \nabla \mathrm{A} \bigotimes$. There were no differences between the numbers of bacteria invading the cells after $6 \mathrm{~h}$ and $8 \mathrm{~h} \nabla \mathrm{A} \backslash$. Similar results were also observed with Streptococcus pyogenes. Streptococcus pyogenes entered the cells $2 \mathrm{~h}$ after adhered to the cells $\triangle \mathrm{B} \otimes$. The maximum invasion was at $6 \mathrm{~h} \otimes \mathrm{B} \otimes$. There were no differences between the numbers of bacteria invading the cells after $6 \mathrm{~h}$ and $8 \mathrm{~h} \otimes \mathrm{B} \otimes .{ }^{*} \mathrm{p}<0.05$. N.S., not significant; CFU, colony-forming units. 

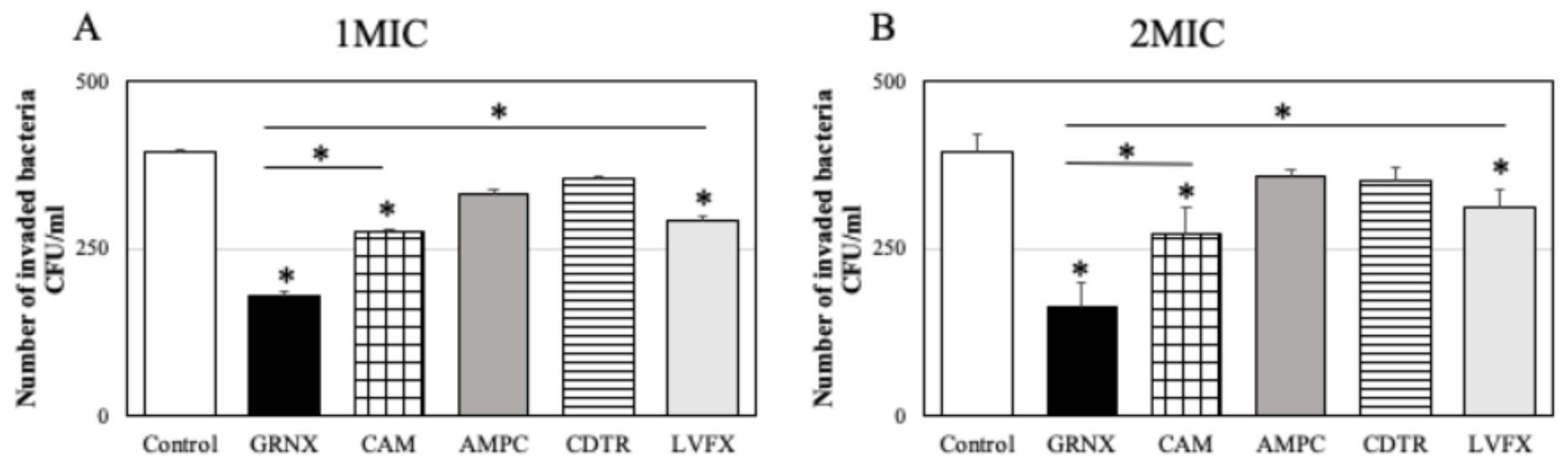

\section{Figure 2}

Effects of antibacterial agents on nontypeable Haemophilus influenzae Cells invaded by bacteria, treated with saponin, and then treated with phosphate-buffered saline (PBS) served as a control. A significant bactericidal effect on NTHi was observed when 1 MIC of GRNX, CAM, or LVFX was used $(A)(p<0.05)$. However, no bactericidal effect was observed from treatment with AMPC or CDTR-PI (A). Similarly, treatment with 2 MIC of GRNX, CAM, or LVFX also had a significant bactericidal effect $(p<0.05)$, but when treated with AMPC or CDTR-PI, no bactericidal effect was observed (B). MIC, minimum inhibitory concentration; NTHi, nontypeable Haemophilus influenzae; GRNX, garenoxacin; CAM, clarithromycin; AMPC, amoxicillin; CDTR-PI, cefditoren pivoxil; LVFX, levofloxacin; N.S., not significant; CFU, colonyforming units.
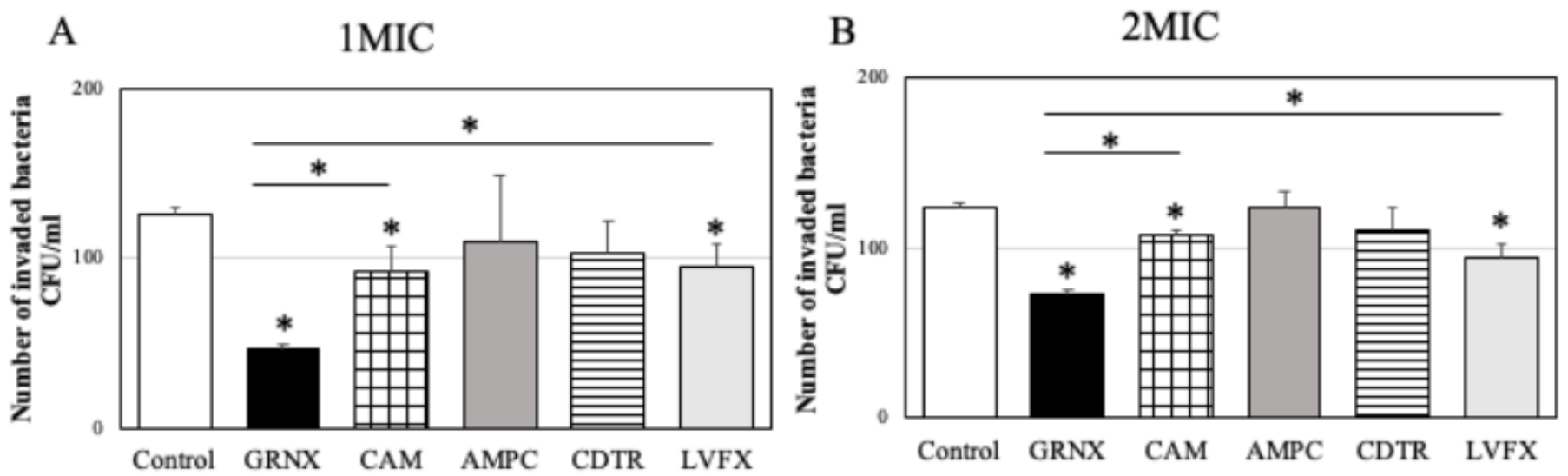

\section{Figure 3}

Effects of antibacterial agents on Streptococcus pyogenes Cells invaded by bacteria, treated with saponin, and then treated with phosphate-buffered saline (PBS) served as a control. A significant 
bactericidal effect on S. pyogenes was observed when 1 MIC of GRNX, CAM, or LVFX was used (A) $(p<$ 0.05). However, no bactericidal effect was observed from treatment with AMPC or CDTR-PI (A). Similarly, treatment with 2 MIC of GRNX, CAM, or LVFX also had a significant bactericidal effect $(p<0.05)$, but when treated with AMPC or CDTR-PI, no bactericidal effect was observed (B). MIC, minimum inhibitory concentration; NTHi, GRNX, garenoxacin; CAM, clarithromycin; AMPC, amoxicillin; CDTR-PI, cefditoren pivoxil; LVFX, levofloxacin; N.S., not significant; CFU, colony-forming units. 